\title{
Population Pharmacokinetics and Pharmacodynamics of Piperaquine in Children With Uncomplicated Falciparum Malaria
}

\author{
J Tarning ${ }^{1,2}$, I Zongo ${ }^{3}$, FA Somé ${ }^{3}$, N Rouamba ${ }^{3}$, S Parikh ${ }^{4}$, PJ Rosenthal ${ }^{4}$, W \\ Hanpithakpong $^{1}$, N Jongrak ${ }^{1}$, NPJ Day ${ }^{1,2}$, NJ White ${ }^{1,2}$, F Nosten $^{1,2,5}$, J-B Ouedraogo $^{3}$, and \\ N Lindegardh ${ }^{1,2}$
}

${ }^{1}$ Mahidol-Oxford Tropical Medicine Research Unit, Faculty of Tropical Medicine, Mahidol University, Bangkok, Thailand ${ }^{2}$ Centre for Tropical Medicine, Nuffield Department of Clinical Medicine, University of Oxford, Oxford, UK ${ }^{3}$ Institut de Recherche en Sciences de la Santé, Bobo-Dioulasso, Burkina Faso ${ }^{4}$ Department of Medicine, University of California, San Francisco, San Francisco, California, USA ${ }^{5}$ Shoklo Malaria Research Unit, Mae Sot, Thailand

\begin{abstract}
Dihydroartemisinin-piperaquine is being increasingly used as a first-line artemisinin combination treatment for malaria. The aim of this study was to describe the pharmacokinetic and pharmacodynamic properties of piperaquine in 236 children with uncomplicated falciparum malaria in Burkina Faso. They received a standard body weight-based oral 3-day fixed-dose dihydroartemisinin-piperaquine regimen. Capillary plasma concentration-time profiles were characterized using nonlinear mixed-effects modeling. The population pharmacokinetics of piperaquine were described accurately by a two-transit-compartment absorption model and a three-compartment distribution model. Body weight was a significant covariate affecting clearance and volume parameters. The individually predicted day 7 capillary plasma concentration of piperaquine was an important predictor $(P<0.0001)$ of recurrent malaria infection after treatment. young children (2-5 years of age) received a significantly higher body weight-normalized dose than older children $(P=0.025)$ but had significantly lower day 7 piperaquine concentrations $(P=$ $0.024)$ and total piperaquine exposures $(P=0.021)$, suggesting that an increased dose regimen for young children should be evaluated.
\end{abstract}

\begin{abstract}
Malaria is one of the world's most important infectious diseases. The worldwide total burden of malaria was estimated to be 225 million cases in 2009, the majority (78\%) of them in Africa. Malaria accounted for an estimated 781,000 deaths during 2009, of which $91 \%$ were in Africa. ${ }^{1}$ Children $<5$ years of age in malaria-endemic countries are especially vulnerable to malaria and to the development of severe disease because they have relatively less immunity than older individuals. Indeed, an estimated $85 \%$ of all deaths associated with malaria occur in children $<5$ years of age. ${ }^{1}$
\end{abstract}

Artemisinin-based combination therapy is now recommended worldwide as first-line treatment for uncomplicated falciparum malaria. One of the most promising new

(C) 2012 American Society for Clinical Pharmacology and Therapeutics

Correspondence: J Tarning (joel@tropmedres.ac).

SUPPLEMENTARY MATERIAL is linked to the online version of the paper at http://www.nature.com/cpt

CONFLICT OF INTEREST The Wellcome Trust is a UK-based medical research charity and is independent of all drug companies. It has no financial links with the manufacturers of either the diagnostic tests or the drugs used in this study. The other authors declared no conflict of interest. 
artemisinin-based combination therapies is the fixed-dose oral combination of dihydroartemisinin and piperaquine, which is now recommended by the World Health Organization (WHO) as a first-line treatment option for uncomplicated falciparum malaria. ${ }^{2}$ High efficacy (PCR-corrected cure rates were 98.7\% (95\% confidence interval (CI): 97.6$99.8 \%$ ) at day 28 ) and good tolerability (e.g., total incidence of early vomiting was only $4.8 \%$ (95\% CI: 3.7-5.9\%)) of dihydroartemisinin-piperaquine were reported in 1,814 adult and pediatric patients with uncomplicated falciparum malaria pooled from 12 different studies in 6 countries between 2003 and 2006. ${ }^{3}$ This pooled analysis indicated that dihydroartemisinin-piperaquine had significantly lower efficacy in children $(n=580)<5$ years of age (PCR-corrected cure rates were $94.2 \%$ (95\% CI: 91.9-96.5\%) at day 28, $P=$ 0.001). In areas endemic for malaria, younger children commonly have lower cure rates than older children and adults because they have less immunity. Although dihydroartemisininpiperaquine was associated with a slightly higher rate of early vomiting, overall it was better tolerated in children than the comparator drugs artemether-lumefantrine, artesunatemefloquine, and amodiaquine-sulfadoxine-pyrimethamine. The fixed dihydroartemisininpiperaquine combination is also one of the most promising alternatives for intermittent preventive treatment in children and pregnant women because it provides long-lasting protective efficacy (because of the long terminal half-life of piperaquine). ${ }^{4,5}$

The pharmacokinetic properties of piperaquine have similarities with those of chloroquine. Studies in rats suggest that renal and biliary clearance of piperaquine are negligible, and it is therefore assumed that piperaquine is eliminated primarily through hepatic metabolism. ${ }^{6,7} \mathrm{In}$ adult patients with uncomplicated falciparum malaria in Southeast Asia, piperaquine displayed a multiphasic distribution with a large apparent volume of distribution $\left(\mathrm{V}_{\mathrm{D}} / \mathrm{F}\right.$ : $574-874$ 1/kg), low oral clearance (CL/F: 0.9-1.4 1/hr/kg), and a consequently long terminal half-life $\left(t_{1 / 2}: 23-28\right.$ days). ${ }^{8} 9$ Somewhat conflicting results have been published regarding the pharmacokinetic properties of piperaquine in children with uncomplicated falciparum malaria. ${ }^{8-10}$ A study in Cambodia by Hung et al., carried out in patients (children as well as adults) with malaria, reported similar values for apparent volume of distribution $(6141 / \mathrm{kg})$ in children $(n=47)$ and in adults $(n=38)$; however, they found a twofold higher oral clearance $(1.85 \mathrm{l} / \mathrm{hr} / \mathrm{kg})$ and a shorter terminal half-life (13.5 days) in the children as compared with the adults. ${ }^{8}$ Karunajeewa et al. reported lower apparent volume of distribution, lower oral clearance, and a shorter terminal elimination half-life in 22 Melanesian children with uncomplicated malaria $\left(\mathrm{V}_{\mathrm{D}} / \mathrm{F}\right.$ of $431 \mathrm{l} / \mathrm{kg}$, CL/F of $0.85 \mathrm{l} / \mathrm{hr} / \mathrm{kg}$, and $t_{1 / 2}$ of 17.2 days) as compared with published results of other studies in adult patients in Southeast Asia. ${ }^{10}$ In a study in Thailand, Tarning et al. suggested that children showed a lower oral piperaquine clearance (CL/F of $0.63 \mathrm{l} / \mathrm{hr} / \mathrm{kg}$ for children between 10 and $20 \mathrm{~kg}$ body weight) and a consequently longer terminal elimination half-life as compared to adults. ${ }^{9}$ However, the authors also stated that the sparse data and the small number of children in the study $(n=11)$ were insufficient to be definitive about these conclusions. Increasingly, concentrations of slowly eliminated antimalarial drugs are measured routinely on day 7 because this reflects the degree of drug exposure that must kill residual malaria parasites for an effective cure. Day 7 values have been used to compare pharmacokinetic properties between populations in whom no pharmacokinetic profile sampling was performed. ${ }^{11,12}$ Low piperaquine concentrations on day 7 are predictive of therapeutic failure; this further supports the rationale for routinely incorporating day 7 measurements into clinical studies. ${ }^{13}$ To date, the pharmacokinetic properties of piperaquine have not been characterized in any African population and the available information in children is limited. The primary objective of this study was to characterize the pharmacokinetic and pharmacodynamic properties of piperaquine in children in Burkina Faso, using nonlinear mixed-effects modeling. 


\section{RESULTS}

A total of 236 children with uncomplicated falciparum malaria were enrolled in this nested pharmacokinetic study in Burkina Faso (demographics shown in Table 1). The fixed oral combination of dihydroartemisinin and piperaquine was well tolerated, with no serious adverse events. The most commonly reported adverse events were early vomiting (within 30 min following drug administration) (5.1\%), headache (5.3\%), late vomiting (6.1\%), and diarrhea (3.4\%).

\section{Pharmacokinetics of piperaquine}

A total of 2,239 capillary plasma samples were obtained after drug administration and the piperaquine concentrations were quantified. Only $13(<1 \%)$ of these samples were below the lower limit of quantification, and these were coded as missing data. The observed piperaquine data was best described by two transit-compartments in the absorption phase followed by three distribution-compartments (Figure 1). ${ }^{14}$ For the distribution phase, a three-compartment distribution model displayed a better fit with observed data than a twocompartment one (difference in objective function value $(\Delta \mathrm{OFV})=-232)$, with no additional benefit of adding another distribution compartment $(\Delta \mathrm{OFV}=-0.075)$. A onecompartment model for distribution showed an OFV that was inferior to those of all other models and was unable to produce convergence. Finally, the observed data were best described in terms of two transit compartments in the absorption phase followed by three distribution compartments, and this was adopted as the base model (Figure 1). The rate constant of molecular transition between the absorption transit compartments was set to be identical so as to obtain a stable model with acceptable precision in parameter estimates. The relative bioavailability $(F)$ was fixed at $100 \%$ but had an allowance for interindividual variability. This approach produced a significantly better fit with observed data $(\triangle \mathrm{OFV}=$ -160 ). An additive error model was adequate to explain the residual random variability, as is expected when logarithmically transformed (LN) heteroscedastic data are modeled. An additive error model on a logarithmic scale is essentially equivalent to an exponential error model on a linear scale. This final base model described the observed data well with no trends in goodness-of-fit diagnostics.

The simultaneous incorporation of body weight as an allometric function on all clearance and volume parameters produced a significantly better fit as compared to the base model $(\triangle \mathrm{OFV}=-45)$, and also decreased the interindividual variability of clearance and volume parameters by up to $34 \%$. Interindividual variability was estimated to be $<1 \%$ for intercompartment clearance $\left(\mathrm{Q}_{1} / \mathrm{F}\right)$ and peripheral volumes of distribution $\left(\mathrm{V}_{\mathrm{P} 1} / \mathrm{F}\right.$ and $\mathrm{V}_{\mathrm{P} 2} /$ F); consequently, the variability component in these parameters could be removed without a significant change in model performance $(\Delta \mathrm{OFV}=-1.87) . \mathrm{Q}_{1} / \mathrm{F}$ and $F$ showed a correlation of $84 \%$ but with an unacceptably low precision for the estimate (relative standard error (RSE) $>85 \%$ ). A model with correlation between $\mathrm{Q}_{1} / \mathrm{F}$ and $F$ also decreased the precision of the random effect estimate in $\mathrm{Q}_{1} / \mathrm{F}(\mathrm{RSE}>60 \%)$ and could not be retained in the model. Continuous and categorical covariates were investigated on the remaining parameters with interindividual variability. The following linear covariate relationships were selected in the forward step $(P<0.05$, in order of inclusion): hemoglobin concentration-oral clearance (CL/ F) $(\triangle \mathrm{OFV}=-9.47)$, body weight $-F(\Delta \mathrm{OFV}=-8.44)$, age-mean transit time $(\mathrm{MTT})$ $(\Delta \mathrm{OFV}=-4.28)$, parasitemia $-\mathrm{CL} / \mathrm{F}(\Delta \mathrm{OFV}=-5.77)$, parasitemia $-F(\Delta \mathrm{OFV}=-4.00)$. None of these covariates could be retained in the backward step with a stricter statistical criterion $(P<0.001)$. The significant covariates in the forward step also had very limited impact on interindividual variability on the specific parameters, and all estimates showed poor precision (RSE $>40 \%$ ). When the model with body weight incorporated as an allometric function on clearance and volume parameters was evaluated, interindividual variability in central volume of distribution $\left(\mathrm{V}_{\mathrm{C}} / \mathrm{F}\right)$ showed an unacceptably low precision 
$(\mathrm{RSE}>75 \%)$ and was therefore removed $(\Delta \mathrm{OFV}=5.17)$. The final parameter estimates are summarized in Table 2.

The final model described the piperaquine data well with no trends in goodness-of-fit plots (Figure 2). Eta shrinkage was computed in the final model: $\mathrm{CL} / \mathrm{F}=27 \%, \mathrm{Q}_{2} / \mathrm{F}=26 \%$, MTT $=41 \%, F=16 \%$, residual random variability $(\sigma)=24 \%$. Epsilon shrinkage was estimated as $7.6 \% .{ }^{15}$ A prediction-corrected visual check $(n=2,000)$ confirmed the final model to have good simulation properties (Figure 3). ${ }^{16}$ A numerical predictive check $(n=2,000)$ calculated 4.8\% (95\% CI: $3.5-6.7 \%$ ) of the observed data to be above the simulated $90 \%$ prediction interval and $4.5 \%$ (95\% CI: 3.5-6.7\%) to be below the simulated $90 \%$ prediction interval.

Although younger children ( $2-5$ years of age, $n=115)$ received a significantly higher body weight-normalized dose of piperaquine $(P=0.025)$ than older children (6-10 years of age, $n$ $=121$ ), the observed day 7 venous and capillary plasma concentrations of piperaquine were significantly lower in the younger children (median (range): 36.6 (6.1-171) vs. 44.1 (14.3141) ng/ml, $P=0.0065$; and 58.7 (17.3-187) vs. 67.5 (28.3-276) ng/ml, $P=0.0098$, respectively). The model predictions were in good agreement with the observed data, resulting in significantly lower predicted day 7 capillary plasma piperaquine concentrations $(P=0.024)$ and total piperaquine exposures $(P=0.021)$ in young children $(2-5$ years $)$ as compared with older children (6-10 years). Body weight and age were highly correlated, and the same pattern could be seen when stratifying predicted day 7 capillary plasma drug concentrations by body weight. This resulted in significantly lower $(P=0.0022)$ concentrations in young children ( $8-17 \mathrm{~kg}$ body weight, $n=115)$ as compared to older children (18-34 kg body weight, $n=121)$, despite the younger children receiving a significantly higher body weight-normalized dose $(P<0.0001)$. It has been proposed in Asia that day 7 venous plasma piperaquine concentration should exceed $30 \mathrm{ng} / \mathrm{ml}$ to ensure therapeutic efficacy. ${ }^{13}$ On the basis of this cutoff value and the correlation between the observed venous and capillary concentrations at day 7 in this study (LN (capillary concentration $)=0.394 \times \mathrm{LN}$ (venous concentration) +2.702 ; data not shown), a corresponding day 7 capillary plasma piperaquine concentration of $\geq 57 \mathrm{ng} / \mathrm{ml}$ was computed as the therapeutic target.

One manufacturer of the fixed oral dihydroartemisinin-piperaquine formulation (Sigma-tau) recommends a body weight-based piperaquine dose regimen of $13-27 \mathrm{mg} / \mathrm{kg} /$ day (Table $3)^{17}$ whereas the WHO recommends a therapeutic dose range of $16-26 \mathrm{mg} / \mathrm{kg} / \mathrm{day}$, with a target dose of $18 \mathrm{mg} / \mathrm{kg} / \mathrm{day}^{2}$ Simulated day 7 capillary concentrations of piperaquine per the WHO and the manufacturer's dosage recommendations resulted in a high proportion of the children having day 7 capillary plasma concentrations below the targeted value of $57 \mathrm{ng} /$ $\mathrm{ml}$ (in children with body weight $8-17 \mathrm{~kg}, 43$ and $45 \%$, respectively; and in those with body weight $18-34 \mathrm{~kg}, 30$ and $27 \%$, respectively) (Table 3, Figure 4). A higher-dose regimen, allowing up to a maximum dose of $30 \mathrm{mg} / \mathrm{kg} /$ day, resulted in $14 \%$ (8-17 $\mathrm{kg}$ body weight) and $18 \%$ (18-34 kg body weight) of simulated patients below the targeted capillary plasma concentrations of piperaquine $(57 \mathrm{ng} / \mathrm{ml})$ at day 7 (Table 3, Figure 4).

\section{Pharmacodynamics of piperaquine}

Two of the children (body weights 10 and $12 \mathrm{~kg}$, with day 7 capillary plasma piperaquine concentrations 37 and $17 \mathrm{ng} / \mathrm{ml}$, respectively) had PCR-confirmed recrudescent falciparum malaria during the 42 days of follow-up. The times to recrudescence were 21 and 35 days, respectively, after the first day of dihydroartemisinin-piperaquine administration. In addition, 25 patients with median (range) day 7 capillary piperaquine concentrations of 49.4 (19.8-72.0) $\mathrm{ng} / \mathrm{ml}$, respectively) had recurrent infections of falciparum malaria (that were classified as new infections by genotyping) at a median (range) time point of 39 (28-42) days after initiation of therapy. A total of five patients were lost to follow-up. Therefore, at 
the end of the follow-up period, the incidences of PCR-confirmed new infections and recrudescent infections were 10.9\% (95\% CI: 7.19-15.7\%) and 0.98\% (95\% CI: $0.12-$ $3.50 \%$ ), respectively. The Cox proportional hazards regression analysis of time elapsed before the occurrence of a new malaria infection displayed the best residual diagnostics with no violation of the proportional hazards assumption $(P=0.20$ for the Schoenfeld's global test). The regression analysis resulted in individually predicted day 7 capillary plasma piperaquine concentrations $(P<0.0001)$, total piperaquine exposure $(P<0.0001)$, and hemoglobin $(P=0.003)$ being independently significant predictors of new malaria infections within the 42-day follow-up period $(n=25)$. Age $(P=0.055)$, body weight $(P=0.058)$, parasitemia $(P=0.27)$, gender $(P=0.10)$, and body temperature $(P=0.14)$ were not significant predictors. Day 7 capillary piperaquine concentrations and piperaquine exposure were highly correlated (data not shown) and could be used interchangeably. No covariate remained a significant predictor in a model containing day 7 capillary piperaquine concentrations, thereby emphasizing the primary importance of antimalarial drug concentrations in suppressing a new malaria infection in this area of high transmission. The regression model resulted in a hazard ratio of 0.941 (95\% CI: $0.920-0.963$ ) for piperaquine capillary concentrations at day 7 , suggesting that the mean risk of acquiring a new malaria infection at any point in the follow-up period increased by $5.9 \%$ for each decrease of $1 \mathrm{ng} /$ $\mathrm{ml}$ in day 7 capillary drug concentrations.

\section{DISCUSSION}

Dihydroartemisinin-piperaquine is one of the most promising artemisinin-based combination therapies in the treatment of uncomplicated falciparum malaria. It has shown good tolerability, no serious adverse effects, and excellent cure rates in the treatment of falciparum malaria in adults. ${ }^{18}$ It is also being evaluated in seasonal malaria chemoprevention in adults and children. ${ }^{3-5,19}$ Several studies have found dihydroartemisinin-piperaquine to be a satisfactory treatment for vivax malaria. ${ }^{20,21}$ Despite its extensive use in the past 10 years, there have been no reported studies on the pharmacokinetics of piperaquine in an African population, and there is little information on the pharmacokinetic properties of the drug in children. ${ }^{8,10}$ Antimalarial dose regimens for children are usually developed empirically from adult data and scaled linearly with body weight for use in a pediatric population. However, important biological processes such as elimination of drugs do not usually scale linearly with body weight, and it is essential that the pharmacokinetics and pharmacodynamics of a treatment are characterized in order to provide an evidence-based dose regimen.

\section{Pharmacokinetics of piperaquine}

The pharmacokinetic properties of piperaquine in this study were best characterized by two transit compartments in the absorption phase followed by three distribution compartments. This novel transit compartment absorption model has not been used in any previous pharmacokinetic model for piperaquine, but it performed well with the study data. The final model allowed for interindividual variability in the mean absorption transit time and relative bioavailability, producing a flexible absorption model with a better fit to the highly variable absorption data. Piperaquine is a lipophilic compound with low oral bioavailability (50\% in rats) and varying total exposure with concomitant food intake. ${ }^{7,22-25}$ Food intake was not controlled for in this study, and this probably explains much of the high intraindividual variability observed in absorption parameters. The distribution pharmacokinetics of piperaquine have been defined previously in terms of a two-compartment model ${ }^{8-10,26}$ It has been suggested that an extra distribution compartment would produce a better fit to observed data, but the amount of data available in those studies could not support the increased number of parameters needed for estimation. ${ }^{9,26}$ Our study confirms that three 
distribution compartments are preferable to a two-compartment model when sufficient data can be incorporated in the model. A previously published analysis of the population pharmacokinetics of piperaquine reported body weight to be a significant linear covariate of elimination clearance and central volume of distribution. ${ }^{9}$ No other studies reported any covariates that explain variability in piperaquine pharmacokinetics. ${ }^{8,10,26}$ Body weight was the only significant covariate in the final model in this study, which is plausible considering the high impact that body weight has on physiologic processes, and the fourfold variation in body weight $(8-34 \mathrm{~kg})$ in the patients enrolled in the study.

The relationship between venous and capillary plasma piperaquine concentrations is variable, and the compartmentalization of piperaquine in different blood cells has a timedependent distribution. ${ }^{27}$ The pharmacokinetic parameter estimates in our study should not, therefore, be compared directly with previously published values, because our study modeled capillary concentration-time data whereas previous studies have all reported pharmacokinetic properties of piperaquine in venous plasma. The pharmacokinetic parameters in our study followed the same patterns as in previously published studies in adult patients, showing large interindividual variability, low elimination clearance, large apparent volume of distribution, and, consequently, a long terminal elimination half-life (Table 2). ${ }^{8,9,28}$ All parameter estimates displayed satisfactory precision and diagnostic performance (Table 2; Figures 2 and 3). Shrinkage in parameter estimates was high, but the calculated epsilon shrinkage was low and should not detract from the reliability of the goodness-of-fit diagnostics. The final model showed good simulation properties, with the 5th, 50th, and 95th percentiles of the observed data contained in the 95\% confidence interval of the Monte Carlo simulations at each respective percentile (Figure 3). Overall, the final model produced robust population pharmacokinetics results and simulations for increased dose regimens.

\section{Pharmacodynamics of piperaquine}

Only two children had PCR-confirmed recrudescent falciparum malaria during the 42 days of follow-up, and their data were not evaluated further. The Cox proportional hazards regression analysis of time elapsed to the occurrence of a new malaria infection $(n=25)$ confirmed that day 7 capillary piperaquine concentration is a good predictor of recurrent malaria infections and that high concentrations delay the inevitable acquisition of new malaria infections in high-transmission settings.

Monte Carlo simulations clearly showed a pattern of lower capillary piperaquine concentrations in young children receiving a body weight-based dosing regimen (Figure 4). Physiological processes do not scale linearly with body weight, and consequently children need a higher body weight-based dose than adults to achieve comparable drug exposure. This has been reported previously for other antimalarial agents such as sulfadoxinepyrimethamine ${ }^{29}$ and is a critical issue in antimalarial treatment guidance, given that young children bear the main brunt of malaria in high-transmission settings. Thus, the manufacturer's dose recommendation for dihydroartemisinin-piperaquine may be too broad, resulting in doses that are too low for children at the low end of $\mathrm{mg} / \mathrm{kg}$ dosing in each dosing group (Figure 4). The current WHO recommendations may also need revision with respect to the dosage of antimalarial drugs to be used in young children with malaria. Simulations suggest that the current recommended therapeutic target of $18 \mathrm{mg} / \mathrm{kg} / \mathrm{day}$ of piperaquine tetra-phosphate is too low for young children ( $8-17 \mathrm{~kg}$ of body weight) because they have a higher body weight-normalized oral clearance. The dose regimen used in this study performed reasonably well and resulted in even concentrations over a broad spectrum of body weights. However, there is still a trend toward low concentrations in many younger children as compared to older ones, and this dose regimen also needs optimization. Based on these findings, an optimized increased dose regimen (Table 3 ) was proposed to ensure that a 
high proportion of children (>25\% at each body weight) had drug levels above the target day 7 capillary plasma piperaquine concentration cutoff of $57 \mathrm{ng} / \mathrm{ml}$. With this, the highest dose regimen would be $30 \mathrm{mg} / \mathrm{kg} /$ day of piperaquine tetra-phosphate and $3.75 \mathrm{mg} / \mathrm{kg} / \mathrm{day}$ of dihydroartemisinin. This increased dose regimen (median (range) dose: $22(18.8-30.0) \mathrm{mg} /$ $\mathrm{kg} / \mathrm{day}$ ) of piperaquine tetra-phosphate was therefore higher than the recommended therapeutic dose target of $18 \mathrm{mg} / \mathrm{kg} / \mathrm{day}$, but for dihydroartemisinin $(2.78(2.33-3.75) \mathrm{mg} /$ $\mathrm{kg} / \mathrm{day}$ ) it was close to the dose target for $4 \mathrm{mg} / \mathrm{kg} /$ day. It could be argued that, because of its excellent efficacy in this study, a dose increase is not needed; however, higher drug levels will reduce the selective pressure for resistance (by preventing under-treatment) and will increase the duration of posttreatment prophylaxis. Dihydroartemisinin-piperaquine is a well tolerated drug at the current dosing level ${ }^{30}$ but no information is available about the safety and tolerability of piperaquine at higher doses. However, the increased dose regimen results in a maximum increase of only $10 \%$ in dihydroartemisinin and piperaquine doses, and this increase would not be expected to reduce tolerability. Dihydroartemisinin has been administered at higher doses ( $4 \mathrm{mg} / \mathrm{kg} /$ day) together with piperaquine $(16 \mathrm{mg} / \mathrm{kg} / \mathrm{day})$ to 241 adult patients with uncomplicated falciparum malaria in Thailand. ${ }^{31,32}$ The dose was well tolerated with no serious adverse events. A formulation of up to $5.3 \mathrm{mg} / \mathrm{kg}$ was well tolerated when administered as a single dose to Thai volunteers but resulted in a significant but transient decrease in hemoglobin and hematocrit. ${ }^{33,34}$ The safety and tolerability of higher doses of dihydroartemisinin and piperaquine must be thoroughly evaluated to support revision in dose recommendations in children. An extrapolation of the model simulations to infants (5-7 kg of body weight) suggests an even greater need for dose adjustment in patients of this body weight group. No data were available in this study for patients with body weights in such a low range, and the issue of optimal dosage in infants requires further investigation.

In conclusion, this study describes the population pharmacokinetic-pharmacodynamic properties of piperaquine in children with malaria in Burkina Faso. The pharmacokinetic properties in this population display trends similar to those in adult populations, and suggest that the day 7 concentration of piperaquine in plasma is an important predictor of therapeutic response. Population simulations suggest that younger children have lower day 7 concentrations than older children when given weight-adjusted dosages, and that an increased dose regimen in younger children is required to ensure adequate drug exposure. The safety and tolerability of a higher-dose regimen in young children should be evaluated.

\section{METHODS}

\section{Study site and ethical approval}

This open-label multicenter pharmacokinetics study was carried out at three public dispensaries for children (Colsama, Ouezzin-Ville, and Sarfalao) in Bobo-Dioulasso, Burkina Faso, from August to December of 2007 (see Supplementary Materials and Methods online). This is an area with a high rate of seasonal malaria transmission from August to October. This study was nested into a larger study of the efficacy and safety of dihydroartemisinin-piperaquine in children, to be reported in full elsewhere (unpublished data). The study was approved by the Committee on Human Research of the University of California, San Francisco, and the Comite d'Ethique Institutionnel du Centre Muraz, BoboDioulasso. The parents or guardians of all the participants gave their informed consent in writing prior to study entry. The trial was registered with Current Controlled Trials (http:// www.controlled-trials.com/ISRCTN59761234). 


\section{Drug regimen}

Patients 2-10 years of age with uncomplicated $P$. falciparum malaria mono-infection received a fixed oral combination of median (range) $2.35(1.88-3.00) \mathrm{mg} / \mathrm{kg} / \mathrm{day}$ of dihydroartemisinin and $18.8(15.0-25.0) \mathrm{mg} / \mathrm{kg} /$ day of piperaquine tetra-phosphate (Duocotexin, Beijing Holley-Cotec Pharmaceuticals, China, dihydroartemisinin $40 \mathrm{mg}$ and piperaquine tetra-phosphate $320 \mathrm{mg}$ ) once a day for 3 days, in accordance with weight-based dosing guidelines ${ }^{2}$ rounded to the nearest quarter tablet. All drug administration was carried out under supervision, and the date and time were recorded. Data from patients in whom a dose was missed were excluded from the pharmacokinetics study. The study drugs given to young children were crushed, mixed with water, and administered as slurry. The study drugs administered to older children were given as tablets or fractions of tablets to be taken orally with a glass of water. Patients were observed for $30 \mathrm{~min}$ after the drug was administered to ensure that the medications were not vomited out. The patients who vomited the medication within 30 min of administration were given an additional dose, but their data were excluded from the pharmacokinetics study.

\section{Pharmacokinetic analysis}

A baseline blood sample was taken before any treatment was given. Capillary blood samples $(\sim 200 \mu \mathrm{l})$ were taken from finger pricks and placed in heparinized tubes on days 2, 3, 4, 5, 7, $14,21,28,35$, and 42 after initial drug administration.

Plasma piperaquine concentrations were determined using solid-phase extraction and liquid chromatography with mass spectroscopy detection, as described previously. ${ }^{35}$ Triplicates of quality-control samples at $4.5,20$, and $400 \mathrm{ng} / \mathrm{ml}$ were used to ensure precision and accuracy during quantification. The coefficients of variation during piperaquine quantification were $4.7,3.7$, and $3.3 \%$ at $4.50,20.0$, and $400 \mathrm{ng} / \mathrm{ml}$, respectively. The lower limit of detection was set to $0.375 \mathrm{ng} / \mathrm{ml}$ and the lower limit of quantification was set to 1.50 $\mathrm{ng} / \mathrm{ml}$.

Piperaquine concentration values in capillary plasma were transformed into their natural logarithms, and piperaquine doses were converted from salt to base form on the basis of molecular weights of piperaquine $(535.5 \mathrm{~g} / \mathrm{mole})$ and piperaquine tetra-phosphate $(927.5 \mathrm{~g} /$ mole). Estimation and simulation of concentration-time data were carried out using a nonlinear mixed-effects approach in NONMEM version 6 (ICON Development Solutions, Hanover, MD) (see Supplementary Materials and Methods online). ${ }^{36}$

\section{Supplementary Material}

Refer to Web version on PubMed Central for supplementary material.

\section{Acknowledgments}

We sincerely thank the study children and their parents/guardians for their cooperation. We also thank the clinical study teams in the dispensaries of Colsama, Sarfalao, and Ouezzin-Ville, and the laboratory technicians, for their assistance. This investigation was supported by Beijing Holley-Cotec Pharmaceuticals and the Doris Duke Charitable Foundation, for which PJR is a Distinguished Clinical Scientist. It was part of the Wellcome TrustMahidol University-Oxford Tropical Medicine Research Program and the PKPDia collaboration, both supported by the Wellcome Trust of Great Britain.

\section{References}

1. Pashynska VA, Van den heuvel H, Claeys M, Kosevich MV. Characterization of noncovalent complexes of antimalarial agents of the artemisinin-type and FE(III)-heme by electrospray mass 
spectrometry and collisional activation tandem mass spectrometry. J. Am. Soc. Mass Spectrom. 2004; 15:1181-1190. [PubMed: 15276165]

2. World Health Organization. Guidelines for the treatment of malaria. 2010.

3. Zwang J, et al. Safety and efficacy of dihydroartemisinin-piperaquine in falciparum malaria: a prospective multi-centre individual patient data analysis. PLoS ONE. 2009; 4:e6358. [PubMed: 19649267]

4. Nankabirwa J, et al. Efficacy, safety, and tolerability of three regimens for prevention of malaria: a randomized, placebo-controlled trial in Ugandan schoolchildren. PLoS ONE. 2010; 5:e13438. [PubMed: 20976051]

5. Bojang KA, et al. Prevention of the recurrence of anaemia in Gambian children following discharge from hospital. PLoS ONE. 2010; 5:e11227. [PubMed: 20574541]

6. Tarning J, et al. Characterization of human urinary metabolites of the antimalarial piperaquine. Drug Metab. Dispos. 2006; 34:2011-2019. [PubMed: 16956956]

7. Tarning J, Lindegardh N, Sandberg S, Day NJ, White NJ, Ashton M. Pharmacokinetics and metabolism of the antimalarial piperaquine after intravenous and oral single doses to the rat. J. Pharm. Sci. 2008; 97:3400-3410. [PubMed: 17969131]

8. Hung TY, et al. Population pharmacokinetics of piperaquine in adults and children with uncomplicated falciparum or vivax malaria. Br. J. Clin. Pharmacol. 2004; 57:253-262. [PubMed: 14998421]

9. Tarning J, et al. Population pharmacokinetics of piperaquine after two different treatment regimens with dihydroartemisinin-piperaquine in patients with Plasmodium falciparum malaria in Thailand. Antimicrob. Agents Chemother. 2008; 52:1052-1061. [PubMed: 18180343]

10. Karunajeewa HA, et al. Pharmacokinetics and efficacy of piperaquine and chloroquine in Melanesian children with uncomplicated malaria. Antimicrob. Agents Chemother. 2008; 52:237243. [PubMed: 17967917]

11. Smithuis F, et al. Efficacy and effectiveness of dihydroartemisinin-piperaquine versus artesunatemefloquine in falciparum malaria: an open-label randomised comparison. Lancet. 2006; 367:2075-2085. [PubMed: 16798391]

12. White NJ, Stepniewska K, Barnes K, Price RN, Simpson J. Simplified antimalarial therapeutic monitoring: using the day-7 drug level? Trends Parasitol. 2008; 24:159-163. [PubMed: 18353727]

13. Price RN, et al. Clinical and pharmacological determinants of the therapeutic response to dihydroartemisinin-piperaquine for drug-resistant malaria. Antimicrob. Agents Chemother. 2007; 51:4090-4097. [PubMed: 17846129]

14. Savic RM, Jonker DM, Kerbusch T, Karlsson MO. Implementation of a transit compartment model for describing drug absorption in pharmacokinetic studies. J. Pharmacokinet. Pharmacodyn. 2007; 34:711-726. [PubMed: 17653836]

15. Savic RM, Karlsson MO. Importance of shrinkage in empirical bayes estimates for diagnostics: problems and solutions. AAPS J. 2009; 11:558-569. [PubMed: 19649712]

16. Bergstrand M, Hooker AC, Wallin JE, Karlsson MO. Prediction-corrected visual predictive checks for diagnosing nonlinear mixed-effects models. AAPS J. 2011; 13:143-151. [PubMed: 21302010]

17. Sigma-tau: Application for Inclusion of Dihydroartemisinin Plus Piperaquine (DHA/PPQ) Fixed Dose combination Tablets in the 17th edition of the WHO Model Lists of Essential Medicines. <http://www.who.int/selection_medicines/committees/expert/18/applications/D_Piperaquine.pdf>

18. Zongo I, et al. Randomized comparison of amodiaquine plus sulfadoxine-pyrimethamine, artemether-lumefantrine, and dihydroartemisinin-piperaquine for the treatment of uncomplicated Plasmodium falciparum malaria in Burkina Faso. Clin. Infect. Dis. 2007; 45:1453-1461. [PubMed: 17990228]

19. Cisse B, et al. Randomized trial of piperaquine with sulfadoxine-pyrimethamine or dihydroartemisinin for malaria intermittent preventive treatment in children. PLoS ONE. 2009; 4:e7164. [PubMed: 19784374]

20. Douglas NM, et al. Plasmodium vivax recurrence following falciparum and mixed species malaria: risk factors and effect of antimalarial kinetics. Clin. Infect. Dis. 2011; 52:612-620. [PubMed: 21292666] 
21. Awab GR, et al. Dihydroartemisinin-piperaquine versus chloroquine to treat vivax malaria in Afghanistan: an open randomized, non-inferiority, trial. Malar. J. 2010; 9:105. [PubMed: 20409302]

22. Hai TN, Hietala SF, Van Huong N, Ashton M. The influence of food on the pharmacokinetics of piperaquine in healthy Vietnamese volunteers. Acta Trop. 2008; 107:145-149. [PubMed: 18585670]

23. Annerberg A, et al. A small amount of fat does not affect piperaquine exposure in patients with malaria. Antimicrob. Agents Chemother. 2011; 55:3971-3976. [PubMed: 21709087]

24. Sim IK, Davis TM, Ilett KF. Effects of a high-fat meal on the relative oral bioavailability of piperaquine. Antimicrob. Agents Chemother. 2005; 49:2407-2411. [PubMed: 15917540]

25. Nguyen TC, Nguyen NQ, Nguyen XT, Bui D, Travers T, Edstein MD. Pharmacokinetics of the antimalarial drug piperaquine in healthy Vietnamese subjects. Am. J. Trop. Med. Hyg. 2008; 79:620-623. [PubMed: 18840754]

26. Röshammar D, Hai TN, Friberg Hietala S, Van Huong N, Ashton M. Pharmacokinetics of piperaquine after repeated oral administration of the antimalarial combination $\mathrm{cV} 8$ in 12 healthy male subjects. Eur. J. Clin. Pharmacol. 2006; 62:335-341. [PubMed: 16570188]

27. Ashley EA, et al. Comparison of plasma, venous and capillary blood levels of piperaquine in patients with uncomplicated falciparum malaria. Eur. J. Clin. Pharmacol. 2010; 66:705-712. [PubMed: 20300743]

28. Nguyen DV, et al. Pharmacokinetics and ex vivo pharmacodynamic antimalarial activity of dihydroartemisinin-piperaquine in patients with uncomplicated falciparum malaria in Vietnam. Antimicrob. Agents Chemother. 2009; 53:3534-3537. [PubMed: 19528277]

29. Barnes KI, Little F, Smith PJ, Evans A, Watkins WM, White NJ. Sulfadoxine-pyrimethamine pharmacokinetics in malaria: pediatric dosing implications. Clin. Pharmacol. Ther. 2006; 80:582596. [PubMed: 17178260]

30. Myint HY, Ashley EA, Day NP, Nosten F, White NJ. Efficacy and safety of dihydroartemisininpiperaquine. Trans. R. Soc. Trop. Med. Hyg. 2007; 101:858-866. [PubMed: 17659311]

31. Ashley EA, et al. Randomized, controlled dose-optimization studies of dihydroartemisininpiperaquine for the treatment of uncomplicated multidrug-resistant falciparum malaria in Thailand. J. Infect. Dis. 2004; 190:1773-1782. [PubMed: 15499533]

32. ICH Expert Working Group. ICH Harmonised Tripartite Guideline, Text on Validation of Analytical Procedures. 1994.

33. Kongpatanakul S, et al. Evaluation of the safety and relative bioavailability of a new dihydroartemisinin tablet formulation in healthy Thai volunteers. Trans. R. Soc. Trop. Med. Hyg. 2007; 101:972-979. [PubMed: 17681360]

34. Kongpatanakul S, Chatsiricharoenkul S, Khuhapinant A, Atipas S, Kaewkungwal J. Comparative study of dihydroartemisinin and artesunate safety in healthy Thai volunteers. Int. J. Clin. Pharmacol. Ther. 2009; 47:579-586. [PubMed: 19761717]

35. Lindegardh N, Annerberg A, White NJ, Day NP. Development and validation of a liquid chromatographic-tandem mass spectrometric method for determination of piperaquine in plasma stable isotope labeled internal standard does not always compensate for matrix effects. J. Chromatogr. B Analyt. Technol. Biomed. Life Sci. 2008; 862:227-236.

36. Beal, SL.; Boeckman, AJ.; Sheiner, LB. NONMEM user's guides. NONMEM Project Group, University of California at San Francisco; 1992. 


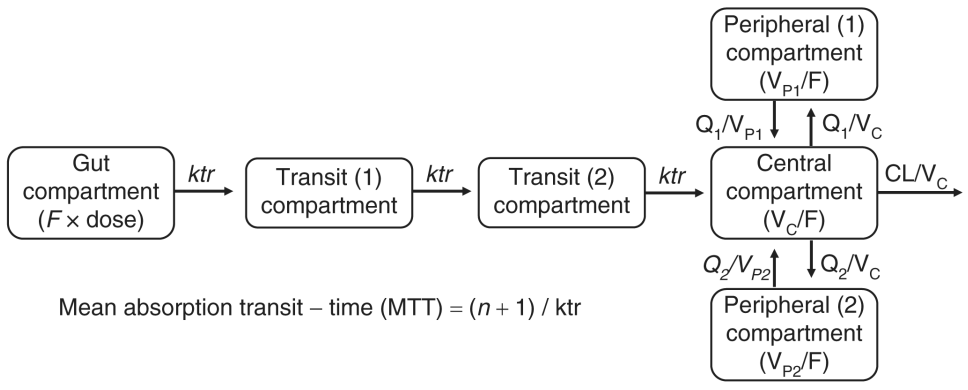

Figure 1.

Final structural model for piperaquine population pharmacokinetics in children with uncomplicated falciparum malaria in Burkina Faso. CL, elimination clearance; $F$, relative oral bioavailability; ktr, transit absorption rate constant; $\mathrm{Q}$, intercompartment clearance; $\mathrm{V}_{\mathrm{C}}$, apparent volume of distribution of the central compartment; $\mathrm{V}_{\mathrm{P}}$, apparent volume of distribution of the peripheral compartment. 

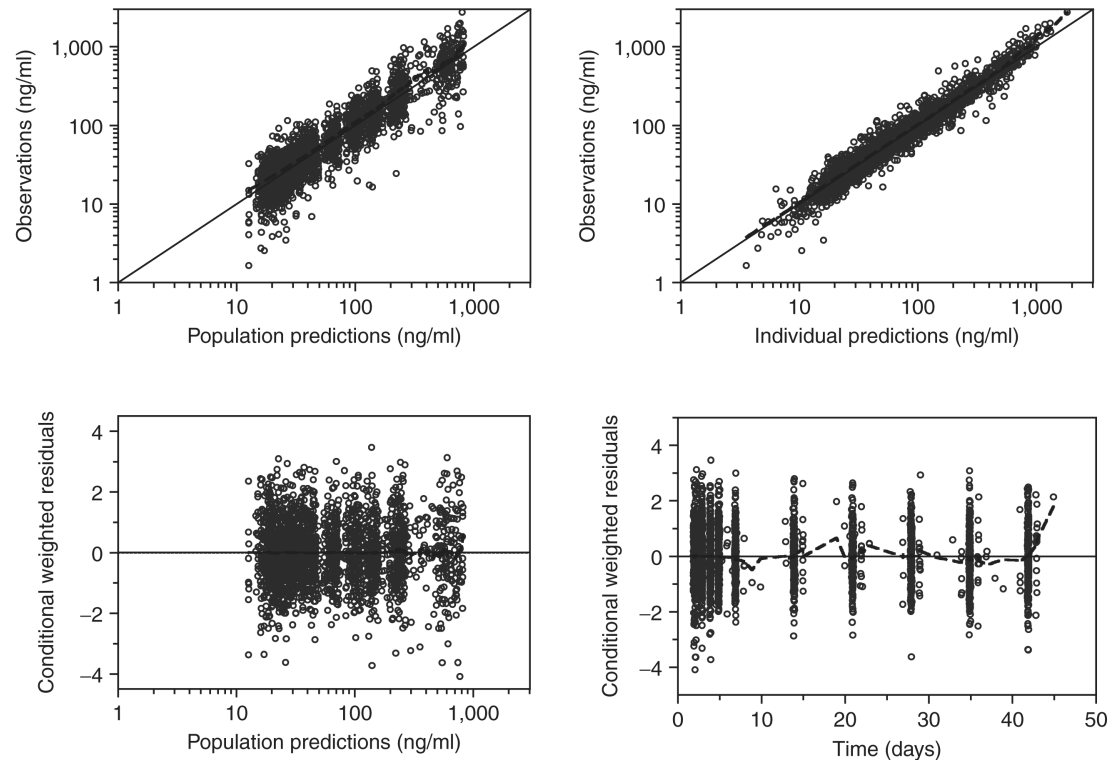

Figure 2.

Goodness-of-fit diagnostics of the final population pharmacokinetics model of piperaquine in children with uncomplicated falciparum malaria in Burkina Faso. Open circles: observed data points; broken line: a locally weighted least-squares regression; solid line: line of identity. The observed capillary plasma piperaquine concentrations, population predictions, and individual predictions were transformed into their logarithms (base 10). 


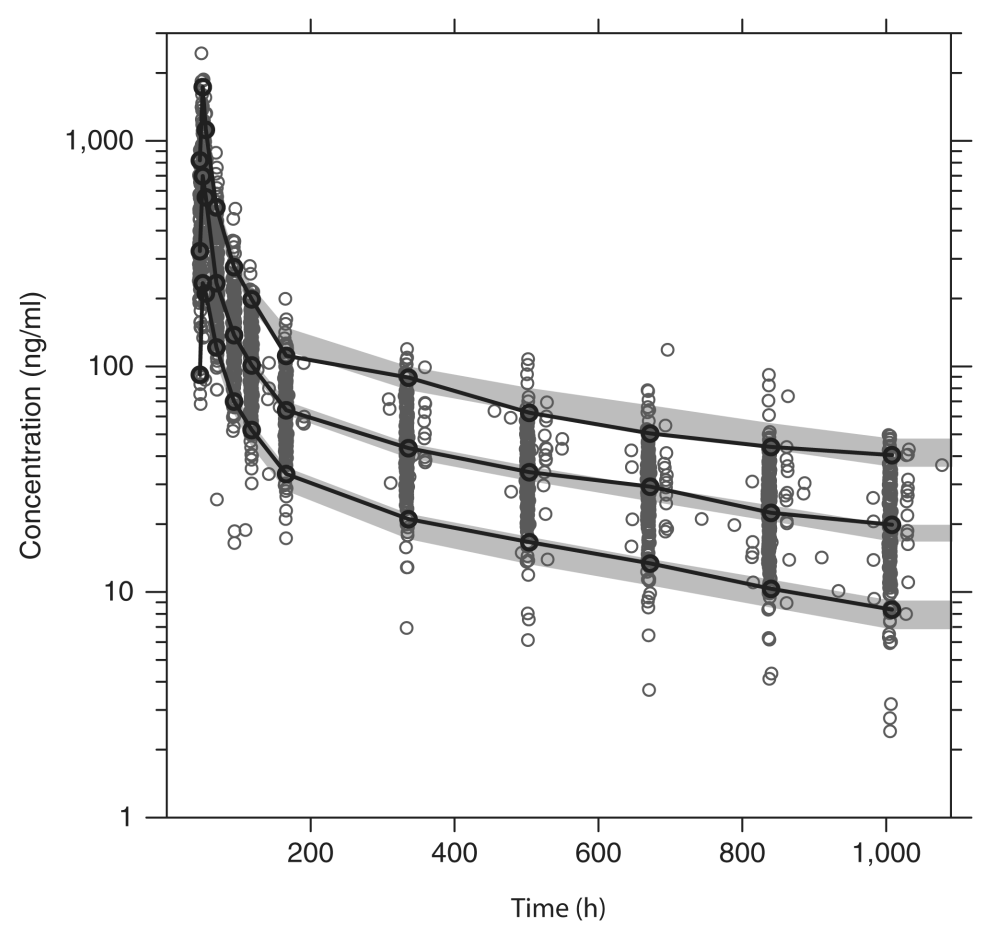

Figure 3.

Prediction-corrected visual predictive check of the final model describing the population pharmacokinetics of piperaquine in children with uncomplicated falciparum malaria in Burkina Faso. Open circles: observed data points; solid lines: 5th, 50th, and 95th percentiles of the observed data; shaded area, 95\% confidence interval of simulated $(n=2,000) 5$ th, 50th, and 95th percentiles. Capillary plasma piperaquine concentrations were transformed into their logarithms (base 10). 

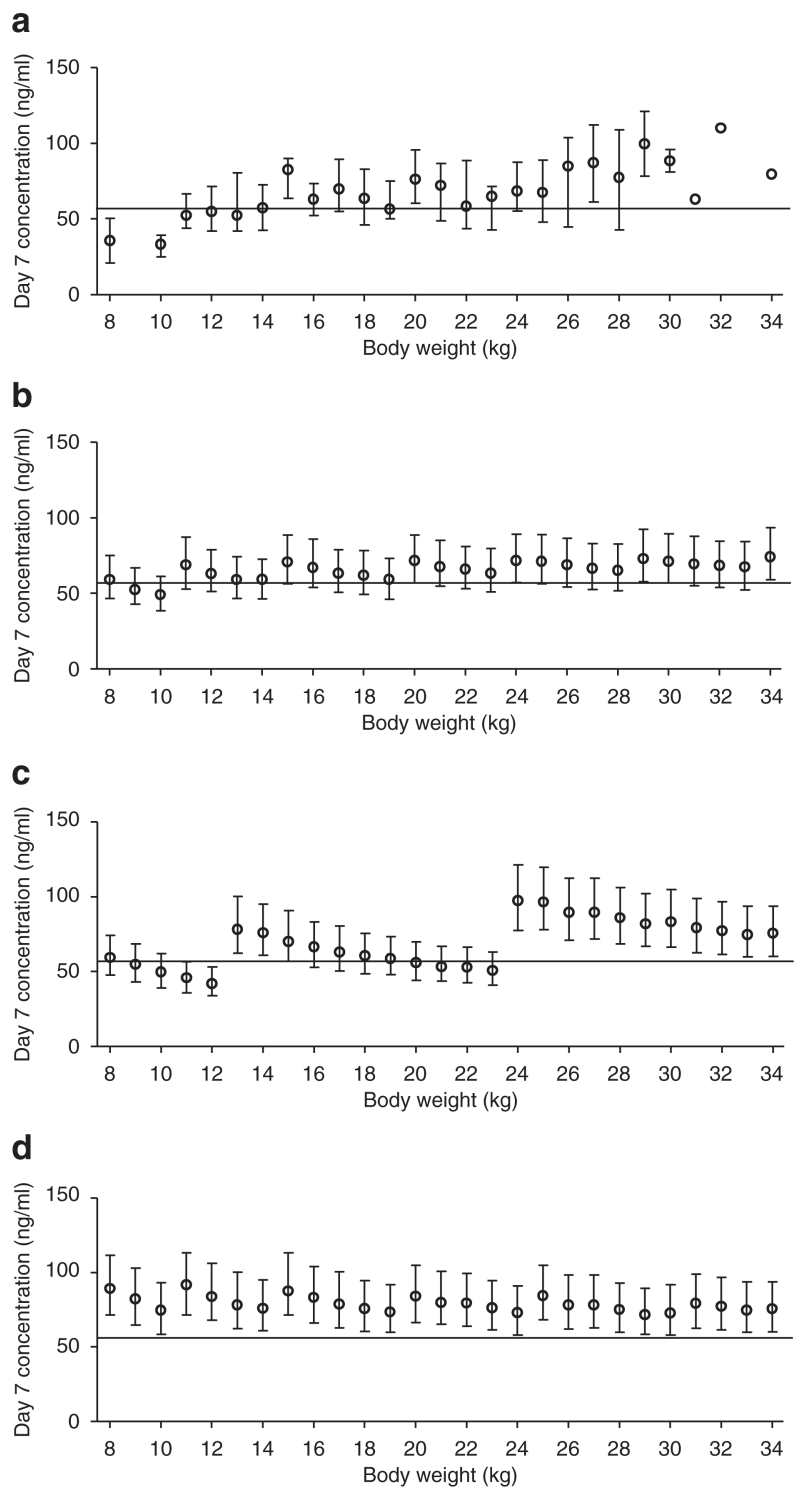

Figure 4.

Observed and simulated day 7 capillary piperaquine concentrations stratified for body weight (1,000 simulated patients at each body weight) after different dose regimens. Black circles: median values \pm interquartile range; black solid horizontal line: cutoff value for therapeutic failure $(57 \mathrm{ng} / \mathrm{ml}$ for capillary concentrations). (a) Observed day 7 capillary concentrations; (b) predicted day 7 capillary concentrations after the dose regimen used in this study; (c) predicted day 7 capillary concentrations after the dose regimen proposed by Sigma-tau; (d) predicted day 7 capillary concentrations after an increased dose regimen to achieve $>75 \%$ of concentrations above the defined cutoff at each body weight (Table 3 ). 
Table 1

Patient demographics, covariates, and treatment outcome in children with uncomplicated falciparum malaria in Burkina Faso

\begin{tabular}{|c|c|}
\hline Total no. of patients & 236 \\
\hline Total no. of samples/patient & $10.0(3.00-11.0)$ \\
\hline $\begin{array}{l}\text { Total dose of piperaquine tetra-phosphate } \\
(\mathrm{mg} / \mathrm{kg})\end{array}$ & $56.5(45.0-72.0)$ \\
\hline Total dose of piperaquine base $(\mathrm{mg} / \mathrm{kg})$ & $32.6(26.1-41.6)$ \\
\hline Total dose of dihydroartemisinin (mg/kg) & $7.06(5.65-9.00)$ \\
\hline \multicolumn{2}{|l|}{ Continuous and categorical covariates } \\
\hline Age (years) & $6(2-10)$ \\
\hline Body weight (kg) & $18(8-34)$ \\
\hline Axillary temperature at admission $\left({ }^{\circ} \mathrm{C}\right)$ & $38.7(36-41)$ \\
\hline Parasitemia at admission (no. of parasites $/ \mu \mathrm{l}$ ) & $30,400(2,000-199,000)$ \\
\hline Hemoglobin at admission (g/l) & $10.6(5.00-15.0)$ \\
\hline Males $(\%)$ & $131(55.5 \%)$ \\
\hline \multicolumn{2}{|l|}{ Treatment outcome ${ }^{a}$} \\
\hline $\begin{array}{l}\text { No. of patients with no parasitemia } \\
\text { reappearance }(\%)\end{array}$ & $204(86.4 \%)$ \\
\hline No. of patients with recrudescence $(\%)$ & $2(0.85 \%)$ \\
\hline Time (days) to recrudescence & $28(21-35)$ \\
\hline No. of patients with new infection (\%) & $25(10.6 \%)$ \\
\hline Time (days) to new infection & $39(28-42)$ \\
\hline No. of patients lost during follow-up (\%) & $5(2.12 \%)$ \\
\hline Time (days) to lost & $7(4-14)$ \\
\hline
\end{tabular}

All values are given as median (range) unless otherwise specified.

${ }^{a}$ As confirmed by PCR. 
Table 2

Parameter estimates of the final model describing piperaquine population pharmacokinetics in children with uncomplicated P. falciparum malaria in Burkina Faso

\begin{tabular}{|c|c|c|c|c|}
\hline & $\begin{array}{c}\text { Population } \\
\text { estimate }^{a} \\
\left(\% \mathrm{RSE}^{b}\right)\end{array}$ & $95 \% \mathrm{CI}^{b}$ & $\begin{array}{c}\text { IIV }(\% \mathrm{CV})^{a} \\
\left(\% \mathrm{RSE}^{b}\right)\end{array}$ & $95 \% \mathrm{CI}^{b}$ \\
\hline \multicolumn{5}{|l|}{ Parameter } \\
\hline CL/F (1/h) & $7.50(4.37)$ & $7.14-7.88$ & $21.0(22.8)$ & $14.3-25.1$ \\
\hline $\mathrm{V}_{\mathrm{C}} / \mathrm{F}(1)$ & $247(14.2)$ & $152-291$ & - & - \\
\hline $\mathrm{Q}_{1} / \mathrm{F}(\mathrm{l} / \mathrm{h})$ & $13.1(30.9)$ & $9.36-27.5$ & - & - \\
\hline $\mathrm{V}_{\mathrm{P} 1} / \mathrm{F}(1)$ & $254(13.3)$ & $215-357$ & - & - \\
\hline $\mathrm{Q}_{2} / \mathrm{F}(\mathrm{l} / \mathrm{h})$ & $10.8(4.59)$ & $9.93-11.8$ & $22.3(25.9)$ & $13.7-27.8$ \\
\hline $\mathrm{V}_{\mathrm{P} 2} / \mathrm{F}(\mathrm{l})$ & $3,340(3.43)$ & $3,140-3,580$ & - & - \\
\hline MTT (h) & $2.15(14.3)$ & $1.75-2.94$ & $79.9(21.8)$ & $62.1-106$ \\
\hline$F(\%)$ & 100 (fixed) & - & $27.6(21.1)$ & $21.9-33.5$ \\
\hline$\sigma$ & $0.264(2.64)$ & $0.251-0.278$ & $19.4(39.8)$ & $9.78-26.6$ \\
\hline \multicolumn{5}{|c|}{ Secondary parameters (median value $($ range $))^{\mathcal{c}}$} \\
\hline $\mathrm{V}_{\mathrm{D}} / \mathrm{F}(1)$ & $3,850(1,710-7,270)$ & - & - & - \\
\hline$t_{1 / 2}$ (days) & $23.2(14.8-31.3)$ & - & - & - \\
\hline $\begin{array}{l}\mathrm{AUC}_{\text {day0-45 }} \\
(\mathrm{h} \times \mu \mathrm{g} / \mathrm{ml})\end{array}$ & $36.4(9.61-93.0)$ & - & - & - \\
\hline$T_{\max }(\mathrm{h})$ & $3.84(1.52-12.4)$ & - & - & - \\
\hline$C_{\max }(\mathrm{ng} / \mathrm{ml})$ & $730(254-1,820)$ & - & - & - \\
\hline $\begin{array}{l}\text { Day } 7 \text { capillary } \\
\text { concentration } \\
(\mathrm{ng} / \mathrm{ml})\end{array}$ & $64.0(16.8-130)$ & - & - & - \\
\hline
\end{tabular}

$\mathrm{AUC}_{\text {day }} 0-45$, area under the capillary plasma concentration-time curve from time point 0 to day $45 ; C_{\text {max }}$, predicted piperaquine capillary peak plasma concentration after last dose; CL, elimination clearance; CL/F, oral clearance; Day 7 capillary plasma concentration, predicted piperaquine capillary concentration at day 7; F, oral bioavailability; MTT, mean absorption transit time; Q1, intercompartment clearance 1; Q2,

intercompartment clearance $2 ; \sigma$, variance of the additive residual error; $T_{\max }$, predicted time to piperaquine capillary peak concentration after last dose; $t_{1 / 2}$, terminal elimination half-life; $V_{C}$, central volume of distribution; $V_{P}$, peripheral volume of distribution $1 ; V_{P}$, peripheral volume of distribution $2 ; \mathrm{V}_{\mathrm{D}}$, apparent total volume of distribution.

${ }^{a}$ Computed population mean values from NONMEM. Interindividual variability (IIV) is calculated as SQRT (EXP(mean value) - 1).

${ }^{b}$ Assessed by nonparametric bootstrap method ( $n=1,000$ iterations) of the final pharmacokinetic model. Population parameter estimates are calculated for a typical patient with a body weight of $18 \mathrm{~kg}$. Relative standard error (RSE) is calculated as $100 \times$ (standard deviation/mean value); $95 \%$ confidence interval $(95 \% \mathrm{CI})$ is displayed as the $2.5-97.5$ percentile of bootstrap estimates.

${ }^{c}$ Population estimates were calculated as median value (range) from empirical Bayes estimates. 
Table 3

Body weight-based treatment guidelines for dihydroartemisinin-piperaquine in children

\begin{tabular}{|c|c|c|c|c|c|c|}
\hline \multirow[b]{2}{*}{ Body weight (kg) } & \multicolumn{2}{|c|}{$\begin{array}{c}\text { Recommended dose regimen (Sigma- } \\
\text { tau) }\end{array}$} & \multicolumn{2}{|c|}{ Study dose regimen } & \multicolumn{2}{|c|}{ Increased dose regimen } \\
\hline & No. of tablets/day & PQP/day (mg/kg) & No. of tablets/day & PQP/day (mg/kg) & No. of tablets/day & PQP/day (mg/kg) \\
\hline 8 & 1 & 20.0 & 1 & 20.0 & 1.5 & 30.0 \\
\hline 9 & 1 & 17.8 & 1 & 17.8 & 1.5 & 26.7 \\
\hline 10 & 1 & 16.0 & 1 & 16.0 & 1.5 & 24.0 \\
\hline 11 & 1 & 14.5 & 1.5 & 21.8 & 2 & 29.1 \\
\hline 12 & 1 & 13.3 & 1.5 & 20.0 & 2 & 26.7 \\
\hline 13 & 2 & 24.6 & 1.5 & 18.5 & 2 & 24.6 \\
\hline 14 & 2 & 22.9 & 1.5 & 17.1 & 2 & 22.9 \\
\hline 15 & 2 & 21.3 & 2 & 21.3 & 2.5 & 26.7 \\
\hline 16 & 2 & 20.0 & 2 & 20.0 & 2.5 & 25.0 \\
\hline 17 & 2 & 18.8 & 2 & 18.8 & 2.5 & 23.5 \\
\hline 18 & 2 & 17.8 & 2 & 17.8 & 2.5 & 22.2 \\
\hline 19 & 2 & 16.8 & 2 & 16.8 & 2.5 & 21.1 \\
\hline 20 & 2 & 16.0 & 2.5 & 20.0 & 3 & 24.0 \\
\hline 21 & 2 & 15.2 & 2.5 & 19.0 & 3 & 22.9 \\
\hline 22 & 2 & 14.5 & 2.5 & 18.2 & 3 & 21.8 \\
\hline 23 & 2 & 13.9 & 2.5 & 17.4 & 3 & 20.9 \\
\hline 24 & 4 & 26.7 & 3 & 20.0 & 3 & 20.0 \\
\hline 25 & 4 & 25.6 & 3 & 19.2 & 3.5 & 22.4 \\
\hline 26 & 4 & 24.6 & 3 & 18.5 & 3.5 & 21.5 \\
\hline 27 & 4 & 23.7 & 3 & 17.8 & 3.5 & 20.7 \\
\hline 28 & 4 & 22.9 & 3 & 17.1 & 3.5 & 20.0 \\
\hline 29 & 4 & 22.1 & 3.5 & 19.3 & 3.5 & 19.3 \\
\hline 30 & 4 & 21.3 & 3.5 & 18.7 & 3.5 & 18.7 \\
\hline 31 & 4 & 20.6 & 3.5 & 18.1 & 4 & 20.6 \\
\hline 32 & 4 & 20.0 & 3.5 & 17.5 & 4 & 20.0 \\
\hline 33 & 4 & 19.4 & 3.5 & 17.0 & 4 & 19.4 \\
\hline 34 & 4 & 18.8 & 4 & 18.8 & 4 & 18.8 \\
\hline
\end{tabular}

One tablet of the fixed dihydroartemisinin-piperaquine pediatric combination contained $20 \mathrm{mg}$ dihydroartemisinin (DHA) and $160 \mathrm{mg}$ piperaquine tetra-phosphate (PQP); $160 \mathrm{mg}$ piperaquine tetra-phosphate corresponds to $92 \mathrm{mg}$ piperaquine base. 